\title{
Sildenafil for pulmonary hypertension complicating idiopathic pulmonary fibrosis: a rationale grounded in basic science
}

\author{
Paul M. Hassoun ${ }^{1}$ and Steven D. Nathan ${ }^{2}$ \\ Affiliations: ${ }^{1}$ Division of Pulmonary and Critical Care Medicine, Dept of Medicine, Johns Hopkins University, \\ Baltimore, MD, USA. ${ }^{2}$ Advanced Lung Disease and Transplant Program, Dept of Medicine, Inova Fairfax
} Hospital, Falls Church, VA, USA.

Correspondence: Paul M. Hassoun, Division of Pulmonary and Critical Care Medicine, Dept of Medicine, Johns Hopkins University, 1830 East Monument Street, Baltimore, MD 21287, USA. E-mail: phassoundjhmi.edu

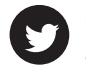

@ERSpublications

Sildenafil may be of benefit in IPF with out-of-proportion PH and could be a step toward effective multimodal therapy http://ow.ly/YYYap

The last few years have seen the development and approval of two drugs, pirfenidone and nintedanib, for the treatment of idiopathic pulmonary fibrosis (IPF) [1-3]. Both agents have been shown to slow the loss of lung function and, therefore, target progressive fibrosis. While the availability of these agents has been a welcome breakthrough, it is well recognised that they both have limited effect, and there remain many unmet needs [4] and a dire necessity for additional therapies $[4,5]$. Aside from the fibrotic component of the disease, another pathophysiological consequence that may supervene in IPF patients is pulmonary hypertension (PH) [6]. $\mathrm{PH}$ complicating the course of patients with pulmonary fibrosis has a strong association with functional impairment and worse outcomes. The likelihood of developing PH increases over time and, in the terminal phases of the disease, most patients have developed an element of $\mathrm{PH}$ [7]. Therefore, in patients with more advanced disease who have established $\mathrm{PH}$ or are at high risk of developing $\mathrm{PH}$, pulmonary vasoactive agents might represent a viable therapeutic option. Targeting PH in IPF makes intuitive sense, but the challenges lie in not only choosing the most appropriate patient population but also the right medication or class of medications.

There are data to suggest that agents targeting the nitric oxide pathway may be best suited to the task $[8,9]$. The Step-IPF study of sildenafil was a negative study based on its primary end-point (proportion of patients with an increase in the 6-min walk distance of $20 \%$ or more) but it did demonstrate significant effects on a number of secondary end-points including quality of life, single-breath diffusing capacity of the lung for carbon monoxide (DLCO) and arterial oxygenation [8]. Based on a subgroup analysis, it appears that those patients with an element of right ventricular dysfunction represent the phenotype most likely to benefit from this therapy [10]. However, one always needs to view subgroup analyses with some circumspection, especially when dealing with small numbers.

In this issue of the European Respiratory Journal, Milara et al. [11] investigated the effects of the phosphodiesterase 5 (PDE5) inhibitor sildenafil on vasoconstriction and remodelling of pulmonary arteries (PAs) from healthy donors and patients with IPF (with or without PH) as well as patients with isolated $\mathrm{PH}$. In separate experiments, the authors examined the effect of sildenafil on ventilation/perfusion $\left(V^{\prime} / Q^{\prime}\right)$ match in a model of bleomycin-induced pulmonary fibrosis. The functional studies related to isolated PA rings were simple and elegant, and included a "relaxant" protocol where cumulative doses of sildenafil were added to PAs pre-contracted with serotonin (5-hydroxytryptamine (5-HT)) and a "contraction" protocol where sildenafil was present in the organ bath before cumulative concentrations of 5-HT were added. In the relaxant protocol, sildenafil was found to induce concentration-dependent relaxation of PA rings mainly in the PAs of control and IPF groups, and to a significantly lesser extent in the PH+IPF and

Received: Feb 222016 | Accepted: Feb 232016

Conflict of interest: Disclosures can be found alongside the online version of this article at erj.ersjournals.com

Copyright @ERS 2016 
PH groups. This effect was only partially dependent on the presence of the endothelium. In the contraction protocol, however, sildenafil had significant effects in PAs from IPF, and more so in PAs from $\mathrm{PH}+\mathrm{IPF}$ and $\mathrm{PH}$, with no effect in PAs from control donors.

These functional differences in PA vasomotor responses prompted further experiments examining markers of cell proliferation and remodelling, and mesenchymal/myofibroblast transition (e.g. smooth muscle $\alpha$-actin, vimentin and transforming growth factor (TGF)- $\beta 1$ expression). In general, these pathways were found to be activated in both IPF and IPF+PH but with more pronounced expression in IPF+PH, indicating a gradual activation of the processes of muscularisation and fibrosis in IPF eventually leading to the PH complication. These results are also consistent with a similar molecular signature obtained with laser capture microdissected PA profiles in combination with whole-genome microarrays from patients with chronic obstructive pulmonary disease and IPF complicated by PH [12]. In addition, Milara et al. [11] found the expression of several endothelial markers (e.g. endothelial nitric oxide synthase, vascular endothelial cadherin and vascular endothelial growth factor receptor) was downregulated in PAs from IPF and IPF+PH, suggestive of endothelial dysfunction. Importantly, PDE5 expression was significantly upregulated in PAs from IPF and $\mathrm{IPF}+\mathrm{PH}$ patients, in strong support of the functional PA ring data. To tie up the results, Milara et al. [11] examined the role of sildenafil and TGF- $\beta$ stimulation on cultured PA ring explants. TGF- $\beta$ was found to decrease the expression of endothelial markers while increasing the expression of PDE5 and mesenchymal markers, effects that were reversed by sildenafil treatment. Similar effects of TGF- $\beta$ and sildenafil were obtained using cultured human PA endothelial and smooth muscle cells obtained from patients with IPF, with sildenafil inhibiting TGF-induced endothelial to mesenchymal and smooth muscle cell to myofibroblast transition, seemingly via inhibition of extracellular signal-regulated kinase $1 / 2$ and SMAD3 phosphorylation.

A significant concern when using any vasodilator drug is the possibility of worsening $V^{\prime} / Q^{\prime}$ matching and, therefore, hypoxaemia. To address this particular issue, Milara et al. [11] tested the effect of sildenafil given by infusion in a model of bleomycin-induced pulmonary fibrosis. While this model may not be ideal for IPF, it is certainly appropriate here for the purpose of testing the effect of vasodilation on $V^{\prime} / Q^{\prime}$ matching. The finding that sildenafil did not in fact alter $V^{\prime} / Q^{\prime}$ matching is in keeping with the results of acute infusion of sildenafil in humans with IPF complicated by $\mathrm{PH}$, which also showed no effect on $V^{\prime} / Q^{\prime}$ [13]. Whether this is the result of sildenafil having anticonstrictive effects while lacking vasodilatory effect (at least in normal arteries), as demonstrated in this study, is an interesting speculation proposed by the investigators. However, it is more likely that sildenafil has vasodilatory effects preferentially in areas that are well ventilated and where nitric oxide is available.

A novelty and strength of this paper relates to the direct study of sildenafil on contractile properties of PAs obtained from patients with IPF with or without $\mathrm{PH}$, and on markers of proliferation and mesenchymal cell transition. The originality of the study is further strengthened by the finding of increased PDE5 expression in PAs from patients with IPF with and without PH, which, to our knowledge, has not been previously described. This important finding then provides a strong rationale for PDE5 as a therapeutic target in IPF complicated by PH.

It is unclear at this time whether PAH specific therapies are of any clinical value in interstitial lung disease complicated by PH. IPF clinical trials using vasodilators such as the endothelin receptor antagonists have thus far been negative [14-16]. It appears, however, that sildenafil has pleiotropic properties beyond its traditional vasodilatory effects that may render it especially attractive as an add-on treatment for IPF. While there have been studies, anecdotal observations and subgroup analyses that have suggested a benefit to sildenafil in IPF, there have also been negative studies that have muted enthusiasm [17, 18]. Interestingly, a recent meta-analysis of 19 randomised clinical trials comparing 10 different interventions (including commonly used $\mathrm{PH}$-specific therapies) with placebo and an average follow-up period of 1 year, without direct comparisons between treatment interventions, suggested that treatment with nintedanib, pirfenidone and sildenafil offered a possible survival advantage in patients with IPF [19].

Sildenafil may ultimately prove to be of benefit in IPF patients with out-of-proportion PH [20] and could be the next "step" forward in the quest toward effective multimodality therapy for IPF. Despite the enticing appeal of sildenafil as a therapy for IPF, there remain many questions that can only be answered in the context of well-planned, randomised, controlled studies [21]. Who should be targeted and at what point in their disease course? How much fibrosis and restrictive disease is permissible when targeting PH? Should the DLCO or echocardiogram suffice as a surrogate or should all trials be based on right heart catheterisation data only? What threshold of PH should be considered before initiating PH-targeted therapy? Should we limit such studies to IPF or be inclusive of all patients with fibrotic lung disease complicated by $\mathrm{PH}$ ? Hopefully, this current paper by Milara et al. [11] will serve as a foundation to engender confidence in this specific therapeutic approach and further ignite interest in ongoing and planned studies targeting the nitric oxide pathway in patients with IPF and other fibrosing lung disorders. 


\section{References}

1 King TE Jr, Bradford WZ, Castro-Bernardini S, et al. A phase 3 trial of pirfenidone in patients with idiopathic pulmonary fibrosis. N Engl J Med 2014; 370: 2083-2092.

2 Richeldi L, du Bois RM, Raghu G, et al. Efficacy and safety of nintedanib in idiopathic pulmonary fibrosis. $N$ Engl J Med 2014; 370: 2071-2082.

3 Noble PW, Albera C, Bradford WZ, et al. Pirfenidone for idiopathic pulmonary fibrosis: analysis of pooled data from three multinational phase 3 trials. Eur Respir J 2016; 47: 243-253.

4 Bonella F, Wijsenbeek M, Molina-Molina M, et al. European IPF Patient Charter: unmet needs and a call to action for healthcare policymakers. Eur Respir J 2016; 47: 597-606.

5 Wuyts WA, Antoniou KM, Borensztajn K, et al. Combination therapy: the future of management for idiopathic pulmonary fibrosis? Lancet Respir Med 2014; 2: 933-942.

6 Nathan SD, Noble PW, Tuder RM. Idiopathic pulmonary fibrosis and pulmonary hypertension: connecting the dots. Am J Respir Crit Care Med 2007; 175: 875-880.

7 Nathan SD, Shlobin OA, Ahmad S, et al. Serial development of pulmonary hypertension in patients with idiopathic pulmonary fibrosis. Respiration 2008; 76: 288-294.

8 Idiopathic Pulmonary Fibrosis Clinical Research Network. A controlled trial of sildenafil in advanced idiopathic pulmonary fibrosis. N Engl J Med 2010; 363: 620-628.

9 Hoeper MM, Halank M, Wilkens H, et al. Riociguat for interstitial lung disease and pulmonary hypertension: a pilot trial. Eur Respir J 2013; 41: 853-860.

10 Han MK, Bach DS, Hagan PG, et al. Sildenafil preserves exercise capacity in patients with idiopathic pulmonary fibrosis and right-sided ventricular dysfunction. Chest 2013; 143: 1699-1708

11 Milara J, Escrivá J, Ortiz JL, et al. Vascular effects of sildenafil in patients with pulmonary fibrosis and pulmonary hypertension: an ex vivo/in vitro study. Eur Respir J 2016; 47: 1737-1749.

12 Hoffmann J, Wilhelm J, Marsh LM, et al. Distinct differences in gene expression patterns in pulmonary arteries of patients with chronic obstructive pulmonary disease and idiopathic pulmonary fibrosis with pulmonary hypertension. Am J Respir Crit Care Med 2014; 190: 98-111.

13 Ghofrani HA, Wiedemann R, Rose F, et al. Sildenafil for treatment of lung fibrosis and pulmonary hypertension: a randomised controlled trial. Lancet 2002; 360: 895-900.

14 Raghu G, Behr J, Brown KK, et al. Treatment of idiopathic pulmonary fibrosis with ambrisentan: a parallel, randomized trial. Ann Intern Med 2013; 158: 641-649.

15 King TE Jr, Brown KK, Raghu G, et al. BUILD-3: a randomized, controlled trial of bosentan in idiopathic pulmonary fibrosis. Am J Respir Crit Care Med 2011; 184: 92-99.

16 Raghu G, Nathan SD, Behr J, et al. Pulmonary hypertension in idiopathic pulmonary fibrosis with mild-to-moderate restriction. Eur Respir J 2015; 46: 1370-1377.

17 Collard HR, Anstrom KJ, Schwarz MI, et al. Sildenafil improves walk distance in idiopathic pulmonary fibrosis Chest 2007; 131: 897-899.

18 Jackson RM, Glassberg MK, Ramos CF, et al. Sildenafil therapy and exercise tolerance in idiopathic pulmonary fibrosis. Lung 2010; 188: 115-123.

19 Rochwerg B, Neupane B, Zhang Y, et al. Treatment of idiopathic pulmonary fibrosis: a network meta-analysis. BMC Med 2016; 14: 18

20 Galiè N, Humbert M, Vachiery JL, et al. 2015 ESC/ERS Guidelines for the diagnosis and treatment of pulmonary hypertension. Eur Respir J 2015; 46: 903-975.

21 Collard HR, Bradford WZ, Cottin V, et al. A new era in idiopathic pulmonary fibrosis: considerations for future clinical trials. Eur Respir J 2015; 46: 243-249. 